\title{
The Effects of Correlated Data and Correction Procedures for F-Test in Unbalanced Two Way Model
}

\author{
Ivan S. Kababchi \\ College of Education \\ University of Salahaddin
}

Received on: $20 / 11 / 2005$

\section{ABSTRACT}

Accepted on: 24/7/2005

Independence of observations is one of the standard assumption in analysis of Variance (ANOVA) table. Where the error terms in the model are independent, identically distributed normal variables with null means and homogeneous variances. In this paper investigate the effect of dependence of observations in ANOVA for unbalanced 2-way nested fixed model and developing a method for adjusting it. When the error terms are correlated and focus on the effects of departures from independence assumptions on hypothesis testing by determining the expect mean squares for errors as well as treatments for this model and correcting the $\mathrm{F}$ statistics for testing the factor effect. The model considered is one in which all measurements have same variance $\sigma^{2}$, and the covariance matrix enjoy a structure defined as follows: every pair of measurements comes from:

i) The same experimental observation and the same experimental unit;

ii) Different experimental observation, but in the same experimental unit;

ii) Different experimental unit;

has covariance $\sigma^{2} \rho_{1}, \sigma^{2} \rho_{2}$ and $\sigma^{2} \rho_{3}$ respectively.

Keywords: analysis of Variance, Independence, distributed normal , correlation , Ftest.

$$
\begin{aligned}
& \text { تأثير البيانات المترابطة وعمليات التصحيح لاختبار - في نموذج غير متوازن ذو اتجاهين } \\
& \text { إيفان س. كبابجي } \\
& \text { كلية التربية ، جامعة صلاح الدين سبان سين }
\end{aligned}
$$

تاريخ القبول: 2005/07/24

تاريخ الاستلام: 2005/11/20

$$
\begin{aligned}
& \text { الملخص } \\
& \text { أن استقلالية المشاهدات تعتبر أحد الفروض الأساسية في جدول تحليل التباين. حيث تتضمن كون حدود }
\end{aligned}
$$

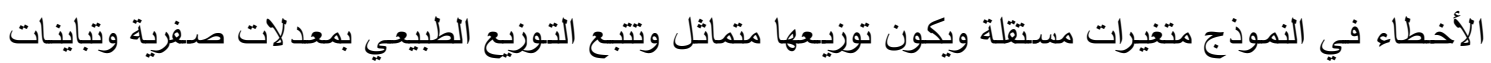

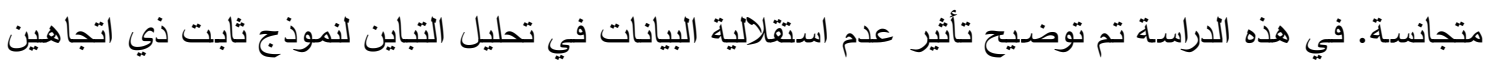

$$
\begin{aligned}
& \text { غير متوازن متشعب كما تم تطوير طريقة لتعديل التباين لهذه المشاهدات المرتبطة. هذا يعني أن حدود الأخطاء } \\
& \text { تكون مترابطة وسنسلط الضوء على تأثير إزالة شرط الاستقلالية على اختبار الفرضيات وذلك بواسطة حساب توقع } \\
& \text { معدل مجموع المربعات للأخطاء، وكذلك للمعالجات في هذا النموذج وتصديح الإحصـاء F لاختبار تأثيرات } \\
& \text { العامل. لهذا نفترض نموذجا جميع القياسـات فيه تمتلك نفس التباين مَّم ومصفوفة التباين المشترك تتمتع ببنية }
\end{aligned}
$$

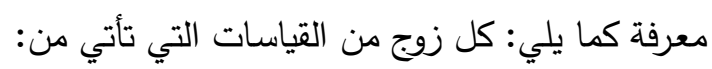

$$
\begin{aligned}
& \text { (1) تكرارات مختلفة ولكن من نفس الوحدة. (2) وحدات جزئية مختلفة بنفس الوحدة. (3) وحدات مختلفة لها } \\
& \text { تباين }
\end{aligned}
$$

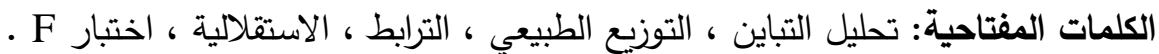




\section{Introduction}

The assumption of independence of observation in ANOVA table may seem like a reasonable assumption in examining data using experimental designs . Rarely is the independent assumption verified in an ANOVA consequently, the analysis of data from experimental design is often hampered by lack of technique to correct the usual F-test for the effect of correlation. Some researches have been devoted to show that slight dependence in the form of serial correlation and interclass correlation can quickly inflate the Type I error rate. See[4], [8], [1] and [3] have shown that in an experimental design, certain forms of dependence can quickly invalidate the result of ANOVA. How small experiment is shown see [6] and see [5] for 2-way corssedand balanced nested classifications respectively.

The aim of this work explains a method for adjusting ANOVA table when observation are correlated, that is, when the error terms are correlated and focus on the effects of departures from independence assumption on hypothesis by determining the expected mean squares for errors as well as treatments for un balanced two way nested fixed effects model (balanced model is special case of un balanced model)and correcting the F statistics for testing the factor effect.

\section{1- Defining the model}

The model of study occurs in ANOVA, when we have a number of independent experimental units and each experimental unit has the same number of experimental observation and each experimental observation receives different number of treatment. See [2], [10], [11].

Let $Y_{i j k}$ be the observation of the kth treatment of the jth experimental observation from the ith experimental unit with $i=1,2, \ldots, \mathrm{m}, j=1,2, \ldots, \mathrm{p}, \mathrm{k}=1,2, \ldots, n_{i j}$

Let

$\mu_{i j k}=E\left(Y_{i j k}\right), \quad e_{i j k}=Y_{i j k}-\mu_{i j k}$

Where $e_{i j k}$ is the error term of the measure $Y_{i j k}$. We assume that the $Y_{i j k}$ distributed normally with mean $\mu_{i j k}$ and that the measurements have the same variance $\sigma^{2}$, and every pair of measurements comes from

i) The same experimental observation and the same experimental unit;

ii) Different experimental observation, but in the same experimental unit;

ii) Different experimental unit;

has covariance $\sigma^{2} \rho_{1}, \sigma^{2} \rho_{2}$ and $\sigma^{2} \rho_{3}$ respectively. Symbolically

$\operatorname{cov}\left(Y_{i j k}, Y_{i^{\prime \prime} k^{\prime}}\right)=\left\{\begin{array}{ccr}\sigma^{2} & \text { if } & i=i^{\prime}, j=j^{\prime}, k=k^{\prime} \\ \sigma^{2} \rho_{1} & \text { if } & i=i^{\prime}, j=j^{\prime}, k \neq k^{\prime} \\ \sigma^{2} \rho_{2} & \text { if } & i=i^{\prime}, j \neq j^{\prime} \\ \sigma^{2} \rho_{3} & \text { if } & i \neq i^{\prime}\end{array}\right.$

In this study, we assume that

$\mu_{i j k}=\theta+\alpha_{i}+\gamma_{i j}$

Where $\theta \in R$ is the overall mean, $\alpha_{i}$ is the effect of the ith experimental unit and $\gamma_{i j}$ is the effect of the ith nested experimental observation in the jth experimental unit; such that 
$\sum_{i} \alpha_{i}=0, \quad \sum_{j} \gamma_{i j}=0 \forall i$

Thus from equations (1) and (3) we have

$Y_{i j k}=\theta+\alpha_{i}+\gamma_{i j}+e_{i j k}$

The model we consider in this part, which is defined by equations (2), (5), is unbalanced 2-way nested effects model. Then from equation (1) the $e_{i j k}$ are distributed normally with mean zero and

$\operatorname{COV}\left(e_{i j k}, e_{i j^{\prime} k^{\prime}}\right)=\operatorname{COV}\left(Y_{i j k}, Y_{i j^{\prime} k^{\prime}}\right)$

First we discuss ANOVA, concerns with the F statistic for the equality of factor level means, and we will discuss correction for correlation.

\section{2- Analysis of Variance}

In this section see [2], [9] and [10] we will partition the total sum of square and degrees of freedom from the model given in equation (5), in the same way that we partition the total sum of square and degrees of freedom in the ANOVA for unbalanced 2-way nested effects model when the observation are independent. Where we partition the total sum of square in the ANOVA table for unbalanced 2-way nested effects model to sum of square of factor A, sum of square of factor B and sum of square of error when the observation are dependent as follows

$$
\begin{aligned}
& S S T O=S S A+S S B+S S e \\
& S S T O=\sum_{i=1}^{m} \sum_{j=1}^{p} \sum_{k=1}^{n_{i j}}\left(Y_{i j k}-\bar{Y}_{\ldots . .}\right)^{2} \\
& S S A=\sum_{i=1}^{m} \sum_{j=1}^{p} n_{i j}\left(\bar{Y}_{i . .}-\bar{Y}_{\ldots}\right)^{2} \\
& S S B=\sum_{i=1}^{m} \sum_{j=1}^{p} n_{i j}\left(\bar{Y}_{i j .}-\bar{Y}_{i . .}\right)^{2} \\
& S S e=\sum_{i=1}^{m} \sum_{j=1}^{p} \sum_{k=1}^{n_{i j}}\left(\bar{Y}_{i j k}-\bar{Y}_{i . .}\right)^{2}
\end{aligned}
$$

The degrees of freedom that corresponds to each of the sums SSA, SSB, SSe, and SSTo are respectively $m-1, m(p-1), m p\left(n_{i j}-1\right), m p N-1$ where $N=n_{11}+n_{12}+n_{13}+\ldots+n_{m p}$. Thus, we will determine the expected mean squares $\mathrm{E}(\mathrm{MS})$. When the observation are not independent by using equation (2) each of the MSSA, MSSB and MSSe.

\subsection{Determining expected mean squares sum of square of error $\mathrm{E}(\mathrm{MSe})$}

Here, we use equation (8d) and the corresponding degrees of freedom for $\mathrm{SSe}$

$M S e=S S e / m p(N-1)$

Now using equation (5) we get

$\bar{Y}_{i j .}=\frac{1}{N} \sum_{j-1}^{n_{i j}}\left(\theta+\alpha_{i}+\gamma_{i j}+e_{i k j}\right)=\theta+\alpha_{i}+\gamma_{i j}+\bar{e}_{i j}$

Where $\bar{Y}_{i j}$. represent the mean of the jth experimental observation; from the ith experimental unit; subtracting equation (10) from equation (5) we get 


$$
Y_{i j k}-\bar{Y}_{i j .}=e_{i j k}-\bar{e}_{i j}
$$

By using equation (8d), (9) and (11) we get

$$
\begin{aligned}
M S e & =\frac{1}{m p(N-1)} \sum_{i=1}^{m} \sum_{j=1}^{p} \sum_{k=1}^{n_{i j}}\left(Y_{i j k}-\bar{Y}_{i j .}\right)^{2} \\
& =\frac{1}{m p(N-1)} \sum_{i=1}^{m} \sum_{j=1}^{p} \sum_{k=1}^{n_{i j}}\left(e_{i j k}-\bar{e}_{i j .}\right)^{2} \\
& =\frac{1}{m p(N-1)} \sum_{i=1}^{m} \sum_{j=1}^{p} \sum_{k=1}^{n_{i j}}\left(e_{i j k}^{2}+\bar{e}_{i j .}{ }^{2}-2 e_{i j k} \bar{e}_{i j .}\right)
\end{aligned}
$$

Now by taking mathematical expectation for (12) we get

$$
E(M S e)=\sum_{i=1}^{m} \sum_{j=1}^{p} \sum_{k=1}^{n_{i j}}\left(A_{1}+A_{2}-2 A_{3}\right)
$$

Where

$$
A_{1}=E\left(e_{i j k}^{2}\right), \quad A_{2}=E\left(\bar{e}_{i j .}^{2}\right), \quad A_{3}=E\left(e_{i j k} \bar{e}_{i j .}\right)
$$

Now using the two equations (1), (2) and (6), we can determine $A_{h}$ for $\mathrm{h}=1,2,3$ as follows

$$
A_{1}=E\left(e_{i j}^{2}\right)=\sigma^{2}
$$

And

$$
\begin{aligned}
A_{3} & =E\left(e_{i j} \bar{e}_{i .}\right)=\frac{1}{n_{i j}} E\left(e_{i j} e_{i .}\right) \\
& =\frac{1}{n_{i j}} E\left(e_{i j}\left(e_{i 1}+e_{i 2}+\cdots+e_{i n_{i}}\right)\right) \\
& =\frac{1}{n_{i j}} E\left(e_{i j}^{2}\right)+\sum_{\substack{j=1 \\
j \neq j^{\prime}}}^{n_{i}} E\left(e_{i j} e_{i j^{\prime}}\right) \\
& =\frac{1}{\mathrm{n}_{\mathrm{ij}}} \sigma^{2}+\left(\mathrm{n}_{\mathrm{ij}}-1\right) \sigma^{2} \rho_{1} \\
& =\frac{\sigma^{2}}{\mathrm{n}_{\mathrm{ij}}}\left(1+n_{i j} \rho_{1}-\rho_{1}\right) \\
& =\frac{\sigma^{2}}{\mathrm{n}_{\mathrm{ij}}}\left(1-\rho_{1}+n_{i j} \rho_{1}\right)
\end{aligned}
$$

\section{And}

$$
\begin{aligned}
A_{2} & =E\left(\bar{e}_{i .}^{2}\right) \\
& =\frac{1}{n_{i}^{2}} E\left(e_{i .}^{2}\right) \\
& =\frac{1}{n_{i}^{2}} E\left(e_{i 1}+e_{i 2}+\ldots+e_{i n_{p}}\right)^{2}
\end{aligned}
$$




$$
\begin{aligned}
& =\frac{1}{\mathrm{n}_{\mathrm{ij}}^{2}}\left[E\left(\sum_{j=1}^{n_{i}} e_{i j}^{2}\right)+E\left(\sum_{j=1}^{n_{i}} \sum_{\substack{j^{\prime}=1 \\
j \neq j^{\prime}}}^{n_{i}} e_{i j} e_{i j^{\prime}}\right)\right] \\
& =\frac{1}{\mathrm{n}_{\mathrm{ij}}^{2}}\left[\sum_{j=1}^{n_{i}} E\left(e_{i j}^{2}\right)+\sum_{\substack{j=1 \\
n_{i}}}^{n_{i}} E\left(e_{i j} e_{i j^{\prime}}\right)\right] \\
& =\frac{1}{j \neq j^{\prime}}\left[n_{\mathrm{ij}}^{2}\left[\sigma_{i} \sigma^{2}+n_{i j}\left(n_{i j}-1\right) \sigma^{2} \rho_{1}\right]\right. \\
& =\frac{\sigma^{2}}{n_{i j}}\left(1-\rho_{1}+n_{j i} \rho_{1}\right)
\end{aligned}
$$

By substituting equation (15a), (15b) and (15c) in equation (13) we get

$$
\begin{aligned}
E(M S e) & =\frac{1}{p(N-1)} \sum_{i=1}^{p} \sum_{j=1}^{n_{i j}}\left(A_{1}-2 A_{2}+A_{3}\right) \\
& =\frac{1}{p(N-1)} \sum_{i=1}^{p} \sum_{j=1}^{n_{i j}}\left[\sigma^{2}-2\left(\frac{\sigma^{2}}{n_{i j}}\left(1-\rho_{1}+n_{i j} \rho_{1}\right)+\frac{\sigma^{2}}{n_{i j}}\left(1-\rho_{1}+n_{i j} \rho_{1}\right)\right]\right. \\
& =\frac{1}{\mathrm{P}(\mathrm{N}-1)} P N\left(\sigma^{2}-\frac{\sigma^{2}}{N}\left(1-\rho_{1}+N \rho_{1}\right)\right. \\
& =\frac{\mathrm{N}}{(\mathrm{N}-1)} \sigma^{2}\left(1-\frac{1}{N}\left(1-\rho_{1}+N \rho\right)\right. \\
& =\frac{\sigma^{2}}{(\mathrm{~N}-1)}(N-1)\left(1-\rho_{1}\right) \\
& =\left(1-\rho_{1}\right) \sigma^{2}
\end{aligned}
$$

\subsection{Determining expected mean squares sum of square of factor B E(MSB)}

Using equation (8c) and the corresponding degrees of freedom for $S S B$

$$
M S B=\frac{S S B}{m(p-1)}
$$

Now using equation (5) we get

$$
\begin{aligned}
\bar{Y}_{i . .} & =\frac{1}{p N} \sum_{j=1}^{p} \sum_{k=1}^{n_{i j}} Y_{i j k} \\
& =\frac{1}{p N} \sum_{j=1}^{p} \sum_{k=1}^{n_{i j}}\left(\theta+\alpha_{i}+\gamma_{i j}+e_{i j k}\right) \\
& =\theta+\alpha_{i}+\bar{e}_{i . .}
\end{aligned}
$$

Subtracting equation (18) from (10) we get

$$
\begin{aligned}
\bar{Y}_{i j .}-\bar{Y}_{i . .} & =\theta+\alpha_{i}+\gamma_{i j}+\bar{e}_{i j .}-\theta-\alpha_{i}-\bar{e}_{. i .} \\
& =\gamma_{i j}+\bar{e}_{i j .}-\bar{e}_{i . .}
\end{aligned}
$$

By using equation (8c), (17) and (19) then 


$$
\begin{aligned}
M S B & =\frac{1}{m(p-1)} \sum_{i=1}^{m} \sum_{j=1}^{p} n_{i j}\left(\bar{Y}_{i j .}-\bar{Y}_{i . .}\right)^{2} \\
& =\frac{1}{m(p-1)} \sum_{i=1}^{m} \sum_{j=1}^{p} n_{i j}\left(\gamma_{i j}+\bar{e}_{i j .}-\bar{e}_{i . .}\right)^{2} \\
& =\frac{1}{m(p-1)} \sum_{i=1}^{m} \sum_{j=1}^{p} n_{i j}\left(\gamma_{i j}{ }^{2}+\bar{e}_{i j .}^{2}+\bar{e}_{i . .}^{2}-2\left(\bar{e}_{i j .} \bar{e}_{i . .}\right)-2\left(\bar{e}_{i j .}-\bar{e}_{i . .}\right)\right)
\end{aligned}
$$

Now by taking the mathematical expectation for equation (20) we get

$$
\begin{aligned}
E(M S B)= & \frac{1}{m(p-1)} \sum_{i=1}^{m} \sum_{j=1}^{p} E\left(n_{i j}\left(\gamma_{i j}^{2}+\bar{e}_{i j .}^{2}+\bar{e}_{i . .}^{2}-2\left(\bar{e}_{i j .} \bar{e}_{i . .}\right)-2 \gamma_{i j}\left(\bar{e}_{i j .}-\bar{e}_{i . .}\right)\right)\right. \\
& =\frac{\sum_{i=1}^{m} \sum_{j=1}^{p} n_{i j} \gamma_{i j}}{m(p-1)}+\frac{1}{m(p-1)} \sum_{i=1}^{m} \sum_{j=1}^{p} n_{i j}\left(B_{1}+B_{2}-2 B_{3}-2 B_{4}\right)
\end{aligned}
$$

Where

$$
B_{1}=E\left(\bar{e}_{i j .}^{2}\right), \mathrm{B}_{2}=E\left(\bar{e}_{\ldots .}^{2}\right), \mathrm{B}_{3}=E\left(\bar{e}_{i j .} \bar{e}_{i . .}\right), \mathrm{B}_{4}=E\left(\bar{e}_{i j .}-\bar{e}_{i . .}\right)
$$

Using equation (2) and (6) we can determine $B_{h}$ for $h=1,2,3,4$ as follows

$$
B_{4}=E\left(\bar{e}_{i j .}-\bar{e}_{i . .}\right)=E\left(\bar{e}_{i j .}\right)-E\left(\bar{e}_{i . .}\right)=0
$$

From equation (15c) we note that $B_{1}=A_{2}$

$$
B_{1}=\frac{\sigma^{2}}{n_{i j}}\left(1-\rho_{1}+n_{j i} \rho_{1}\right)
$$

And

$$
\begin{aligned}
B_{2}= & E\left(\bar{e}_{i . .}^{2}\right)=\frac{1}{p^{2} n_{i j}^{2}} E\left(e_{i . .}^{2}\right) \\
= & \frac{1}{p^{2} n_{i j}^{2}} E\left(e_{i 11}+e_{i 12}+\ldots+e_{i 1 n_{i 1}}+e_{i 11}+e_{211}+e_{i 22}+\ldots\right. \\
& \left.\quad+e_{i 2 n_{i 2}}+e_{i j 1}+e_{i j 2}+\ldots+e_{i j n_{i j}}+\ldots+e_{i p 1}+e_{i p 2}+\ldots+e_{i p n_{i p}}\right)^{2} \\
= & \frac{1}{p^{2} n_{i j}^{2}} E\left(\sum_{j=1}^{p} \sum_{k=1}^{n_{i j}} e_{i j k}^{2}+\sum_{k=1}^{p} \sum_{\substack{k, k^{\prime}=1 \\
k \neq k^{\prime}}}^{n_{i j}} e_{i j k} e_{i j^{\prime} k^{\prime}}+\sum_{\substack{j=1 \\
j \neq j^{\prime}}}^{p} \sum_{k, k^{\prime}}^{n_{i j}} e_{i j k} e_{i j^{\prime} k^{\prime}}\right) \\
= & \frac{1}{p^{2} n_{i j}^{2}}\left(\sum_{j=1}^{p} \sum_{k=1}^{n_{i j}} E\left(e_{i j k}^{2}\right)+\sum_{k=1}^{p} \sum_{\substack{k, k^{\prime}=1 \\
k \neq k^{\prime}}}^{n_{i j}} E\left(e_{i j k} e_{i j j^{\prime} k^{\prime}}\right)+\sum_{\substack{j=1 \\
j \neq j^{\prime}}}^{p} \sum_{k, k^{\prime}}^{n_{i j}} E\left(e_{i j k} e_{i j j^{\prime} k^{\prime}}\right)\right) \\
= & \frac{1}{p^{2} n_{i j}^{2}}\left(p n_{i j} \sigma^{2}+p n_{i j}\left(n_{i j}-1\right) \sigma^{2} \rho_{1}+n_{i j}^{2}(p-1) \rho_{2}\right) \\
= & \frac{\sigma^{2}}{p n_{i j}}\left(1+\left(n_{i}-1\right) \rho_{1}+(p-1) n_{i j} \rho_{2}\right)
\end{aligned}
$$




$$
=\frac{\sigma^{2}}{p n_{i j}}\left(1-\rho_{1}+n_{i j}\left(\rho_{1}-\rho_{2}\right)+n_{i j} p \rho_{2}\right)
$$

And

$$
\begin{aligned}
& B_{3}=E\left(\bar{e}_{i j .} \bar{e}_{i . .}\right)=\frac{1}{p n_{i j}^{2}} E\left(e_{i j .} e_{i . .}\right) \\
& =\frac{1}{p n_{i j}^{2}} E\left(( e _ { i j 1 } + e _ { i j 2 } + \ldots + e _ { i j n _ { i j } } ) \left(e_{i 11}+e_{i 12}+\ldots+e_{i 1 n_{i 1}} e_{i 21}+e_{i 22}+\right.\right. \\
& \left.\left.\ldots+e_{i 2 n_{i 2}}+e_{1 i j 1}+e_{i j 2}+\ldots+e_{i j n_{i j}}+e_{i p 1}+e_{i p 2}+\ldots+e_{i p n_{i p}}\right)\right) \\
& =\frac{1}{p n_{i j}^{2}} E\left[\sum_{k=1}^{n_{i j}} e_{i j k}^{2}+\sum_{\substack{k, k^{\prime}=1 \\
k \neq k^{\prime}}}^{n_{i j}} e_{i j k} e_{i i j k^{\prime}}+\sum_{\substack{j=1 \\
j \neq j^{\prime}}}^{p} \sum_{i k=1}^{n_{i j}} \sum_{k^{\prime}=1}^{n_{i j}} e_{i j k} e_{i j^{\prime} k^{\prime}}\right] \\
& =\frac{1}{p n_{i j}^{2}}\left[\sum_{k=1}^{n_{i j}} E\left(e_{i j k}^{2}\right)+\sum_{\substack{k, k^{\prime}=1 \\
k \neq k^{\prime}}}^{n_{i j}}\left(e_{i j k} e_{i i j k^{\prime}}\right)+\sum_{\substack{j=1 \\
j \neq j^{\prime}}}^{p} \sum_{i k=1}^{n_{i j}} \sum_{k^{\prime}=1}^{n_{i j}}\left(e_{i j k} e_{i j^{\prime} k^{\prime}}\right)\right] \\
& =\frac{1}{p n_{i j}^{2}}\left[n_{i j} \sigma^{2}+n_{i j}\left(n_{i j}-1\right) \rho_{1} \sigma^{2}+n_{i j}^{2}(p-1) \rho_{2} \sigma^{2}\right] \\
& =\frac{\sigma^{2}}{p n_{i j}}\left[1+\left(n_{i j}-1\right) \rho_{1}+n_{i j}(p-1) \rho_{2}\right] \\
& =\frac{\sigma^{2}}{p n_{i j}}\left[1-\rho_{1}+n_{i j}\left(\rho_{1}-\rho_{2}\right)+p n_{i j} \rho_{2}\right]
\end{aligned}
$$

Substituting equation (23a), (23b), (23c) And (23d) in equation (21) we get

$$
\begin{aligned}
& E(M S B)=\frac{\sum_{i=1}^{m} \sum_{j=1}^{p} n_{i j} \gamma_{i j}}{m(p-1)}+\frac{1}{m(p-1)} \sum_{i=1}^{m} \sum_{j=1}^{p} n_{i j}\left(B_{1}+B_{2}-2 B_{3}-2 B_{4}\right) \\
& \left.=\frac{\sum_{i=1}^{m} \sum_{j=1}^{p} n_{i j} \gamma_{i j}}{m(p-1)}+\frac{1}{m(p-1)} \sum_{i=1}^{m} \sum_{j=1}^{p} n_{i j}\left[\frac{\sigma^{2}}{n_{i j}}\left(1-\rho_{1}+n_{i j} \rho_{1}\right)+\frac{\sigma^{2}}{p n_{i j}}\left(1-\rho_{1}\right)+n_{i j}\left(\rho_{1}-\rho_{2}\right)+p n_{i j} \rho_{2}\right)+\frac{\sigma^{2}}{p n_{i j}}\left(1-\rho_{1}+n_{i j}\left(\rho_{1}-\rho_{2}\right)+p n_{i j} \rho_{2}\right)\right] \\
& =\frac{\sum_{i=1}^{m} \sum_{j=1}^{p} n_{i j} \gamma_{i j}}{m(p-1)}+\frac{\sigma^{2}}{m(p-1)}\left[m p\left(1-\rho_{1}\right)+\sum_{i=1}^{m} \sum_{j=1}^{p} n_{i j} \rho_{1}-m\left(1-\rho_{1}\right)-\sum_{i=1}^{m} \sum_{j=1}^{p} \frac{n_{i j}}{p}\left(\rho_{1}-\rho_{2}\right)-\sum_{i=1}^{m} \sum_{j=1}^{p} n_{i j} \rho_{2}\right] \\
& =\frac{\sum_{i=1}^{m} \sum_{j=1}^{p} n_{i j} \gamma_{i j}}{m(p-1)}+\frac{\sigma^{2}}{m(p-1)}\left[m p(p-1)\left(1-\rho_{1}\right)+\sum_{i=1}^{m} \sum_{j=1}^{p} n_{i j}\left(\rho_{1}-\rho_{2}\right)-\sum_{i=1}^{m} \sum_{j=1}^{p} \frac{n_{i j}}{p}\left(\rho_{1}-\rho_{2}\right)\right] \\
& =\frac{\sum_{i=1}^{m} \sum_{j=1}^{p} n_{i} \gamma_{i j}}{m(p-1)}+\frac{\sigma^{2}}{m(p-1)}\left[m p(p-1)\left(1-\rho_{1}\right)+\left(1-\frac{1}{p}\right) \sum_{i=1}^{m} \sum_{j=1}^{p} n_{i j}\left(\rho_{1}-\rho_{2}\right)\right] \\
& =\frac{\sum_{i=1}^{m} \sum_{j=1}^{p} n_{i j} \gamma_{i j}}{m(p-1)}+\sigma^{2}\left[\left(1-\rho_{1}\right)+\frac{1}{m p} \sum_{i=1}^{m} \sum_{j=1}^{p} n_{i j}\left(\rho_{1}-\rho_{2}\right)\right]
\end{aligned}
$$




\subsection{Determining expected mean squares sum of square of factor A E(MSA)}

Using equation (8d) and the corresponding degrees of freedom for $S S A$

$$
M S A=\frac{S S A}{(m-1)}
$$

Now using equation (5) we get

$$
\begin{aligned}
\bar{Y}_{\ldots} & =\frac{1}{m p N} \sum_{i=1}^{m} \sum_{j=1}^{p} \sum_{k=1}^{n_{i j}} Y_{i j k} \\
& =\frac{1}{p N} \sum_{j=1}^{p} \sum_{k=1}^{n_{i j}}\left(\theta+\alpha_{i}+\gamma_{i j}+e_{i j k}\right) \\
& =\theta+\bar{e}^{. .}
\end{aligned}
$$

Subtracting equation (26) from (10) we get

$$
\begin{aligned}
\bar{Y}_{i . .}-\bar{Y}_{. . .} & =\theta+\alpha_{i}+\bar{e}_{i . .}-\theta-\bar{e}_{\ldots} \\
& =\alpha_{i}+\bar{e}_{i . .}-\bar{e}_{\ldots .}
\end{aligned}
$$

By using equation (8),(25)and (27) then

$$
\begin{aligned}
M S A & =\frac{1}{(m-1)} \sum_{i=1}^{m} \sum_{j=1}^{p} \sum_{k=1}^{n_{i j}}\left(\bar{Y}_{i . .}-\bar{Y}_{\ldots}\right)^{2} \\
& =\frac{1}{(m-1)} \sum_{i=1}^{m} \sum_{j=1}^{p} \sum_{k=1}^{n_{i j}}\left(\alpha_{i}+\bar{e}_{i j .}-\bar{e}_{i . .}\right)^{2} \\
& =\frac{1}{(m-1)} \sum_{i=1}^{m} \sum_{j=1}^{p} \sum_{k=1}^{n_{i j}}\left(\alpha_{i}^{2}+\bar{e}_{i . .}^{2}+\bar{e}_{\ldots}^{2}-2\left(\bar{e}_{i . .} \bar{e}_{\ldots}\right)-2 \alpha\left(\bar{e}_{i . .}-\bar{e}_{\ldots}\right)\right)
\end{aligned}
$$

Now by taking the mathematical expectation for equation (20) we get

$$
\begin{aligned}
E(M S A)= & \frac{1}{(m-1)} \sum_{i=1}^{m} \sum_{j=1}^{p} \sum_{k=1}^{n_{i j}} E\left(\alpha_{i}^{2}+\bar{e}_{i . .}^{2}+\bar{e}_{\ldots}^{2}-2\left(\bar{e}_{i . .} \bar{e}_{\ldots}\right)-2 \alpha\left(\bar{e}_{i . .}-\bar{e}_{. . .}\right)\right) \\
= & \left.\frac{\sum_{i=1}^{m} \sum_{j=1}^{p} n_{i j} \alpha_{i}}{(m-1)}+\frac{1}{(m-1)} \sum_{i=1}^{m} \sum_{j=1}^{p} \sum_{k=1}^{n_{i j}} E\left(\bar{e}_{i i . .}^{2}\right)+E\left(\bar{e}_{\ldots}^{2}\right)-2 E\left(\bar{e}_{i . .} \bar{e}_{\ldots}\right)-2 \alpha E\left(\bar{e}_{i . .}-\bar{e}_{\ldots}\right)\right) \\
= & \frac{\sum_{i=1}^{m} \sum_{j=1}^{p} n_{i j} \alpha_{i}}{(m-1)}+\frac{1}{(m-1)} \sum_{i=1}^{m} \sum_{j=1}^{p} \sum_{k=1}^{n_{i j}}\left(C_{1}+C_{2}-2 C_{3}-2 \alpha_{i} C_{3}\right)
\end{aligned}
$$

Where

$$
C_{1}=E\left(\bar{e}_{i . .}^{2}\right), \mathrm{C}_{2}=E\left(\bar{e}_{\ldots . .}^{2}\right), \mathrm{C}_{3}=E\left(\bar{e}_{i . . .} \bar{e}_{i . . .}\right), \mathrm{C}_{4}=E\left(\bar{e}_{i . .}-\bar{e}_{\ldots}\right)
$$

Using equation (2) and (6)we can determine $B_{h}$ for $h=1,2,3,4$ as follows

$C_{4}=E\left(\bar{e}_{i_{. .}}-\overline{e_{i}}\right)=E\left(\bar{e}_{i . .}\right)-E(\bar{e})=0$

From equation (15c) we note that $C_{1}=B_{2}$

$$
\left.C_{1}=\frac{\sigma^{2}}{p n_{i j}}\left[\left(1-\rho_{1}\right)+n_{j i}\left(\rho_{1}-\rho_{2}\right)+p n_{j i} \rho_{2}\right)\right]
$$


And

$$
\begin{aligned}
& C_{2}=E\left(\bar{e}_{\ldots}^{2}\right)=\frac{1}{m^{2} p^{2} n_{i j}^{2}} E\left(e_{\ldots}^{2}\right) \\
& =\frac{1}{\mathrm{p}^{2} n_{i j}^{2}} \mathrm{E}\left(e_{111}+e_{112}+\ldots+e_{11 n_{11}}+\ldots+e_{i j 1}+e_{i j 2}+\ldots+e_{i j n_{i j}}+\ldots+e_{1 p 1}+e_{1 p 2}+\ldots+e_{1 p n_{1 p}}\right. \\
& \left.+\ldots+e_{i p 1}+e_{i p 2}+\ldots+e_{i p n_{i p}}+\ldots+e_{m p 1}+e_{m p 2}+\ldots+e_{m p n_{m p}}\right)^{2}
\end{aligned}
$$

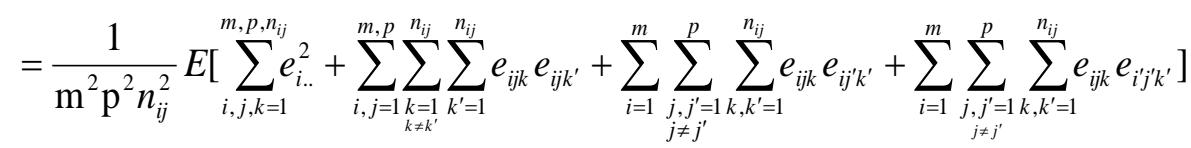

$$
\begin{aligned}
& =\frac{1}{\mathrm{~m}^{2} \mathrm{p}^{2} n_{i j}^{2}}\left[\sum_{i, j, k=1}^{m, p, n_{i j}} E\left(e_{i . .}^{2}\right)+\sum_{i, j=1}^{m, p} \sum_{\substack{k=1 \\
k \neq k^{\prime}}}^{n_{i j}} \sum_{k^{\prime}=1}^{n_{i j}} E\left(e_{i j k} e_{i j k^{\prime}}\right)+\sum_{i=1}^{m} \sum_{\substack{j, j^{\prime}=1 \\
j \neq j^{\prime}}}^{p} \sum_{k, k^{\prime}=1}^{n_{i j}} E\left(e_{i j k} e_{i j^{\prime} k^{\prime}}\right)+\sum_{i=1}^{m} \sum_{\substack{j, j^{\prime}=1 \\
j \neq j^{\prime}}}^{p} \sum_{k, k^{\prime}=1}^{n_{i j}} E\left(e_{i j k} e_{i j^{\prime} k^{\prime}}\right)\right] \\
& =\frac{1}{m^{2} p^{2} n_{i j}^{2}}\left(m p n_{i j} \sigma^{2}+m p n_{i j}\left(n_{i j}-1\right) \sigma^{2} \rho_{1}+m p(p-1) n_{i j}^{2} \rho_{2}+m(m-1) p^{2} n_{i j}^{2} \rho_{3}\right) \\
& =\frac{\sigma^{2}}{m p n_{i j}}\left(1+n_{i j}\left(\rho_{1}-\rho_{2}\right)+n_{i j}\left(\rho_{2}-\rho_{3}\right)+m p n_{i j} \rho_{3}-\rho_{1}\right)
\end{aligned}
$$

And

$$
\begin{aligned}
C_{3}= & E\left(\bar{e}_{i . . .} \bar{e}_{e}\right)=\frac{1}{m p^{2} n_{i j}} E\left(e_{i . .} e_{\ldots}\right) \\
= & \frac{1}{m p^{2} n_{i j}} E\left(\left(e_{i 11}+e_{i 12}+\ldots+e_{i 1 n_{i 1}}+\ldots+e_{i j 1}+e_{i j 2}+\ldots+e_{i j n_{i j}}+\ldots+e_{i p 1}+e_{i p 2}+\ldots+e_{i p n_{i p}}\right)\right. \\
& \left(e_{111}+e_{112}+\ldots+e_{11 n_{11}}+\ldots+e_{i j 1}+e_{i j 2}+\ldots+e_{i j n_{i j}}+\ldots+e_{1 p 1}+e_{1 p 2}+\ldots+e_{1 p n_{1 p}}+\ldots+e_{i j 1}+\right. \\
& \left.\left.e_{i j 2}+\ldots+e_{i j n_{i j}}+\ldots+e_{m p 1}+e_{m p 2}+\ldots+e_{m p n_{m p}}\right)\right) \\
\mathrm{C}_{3}= & \frac{1}{\mathrm{mp}^{2} n_{i j}^{2}} E\left[\sum_{j, k=1}^{p, n_{i j}} e_{i j k}^{2}+\sum_{j=1}^{p} \sum_{k, k^{\prime}=1}^{n_{i j}} e_{i j k} e_{i j^{\prime} k^{\prime}}+\sum_{j, j^{\prime}=1}^{p} \sum_{k, k^{\prime}=1}^{n_{i j}} e_{i j k} e_{i j^{\prime} k^{\prime}}+\sum_{\substack{i=1 \\
i \neq i}}^{m} \sum_{j, j^{\prime}=1}^{p} \sum_{k, k^{\prime}=1}^{n_{i j}} e_{i j k} e_{i j^{\prime} k^{\prime} k^{\prime}}\right] \\
= & \frac{1}{\mathrm{mp}^{2} n_{i j}^{2}}\left[\sum_{j, k=1}^{p, n_{i j}} E\left(e_{i j k}^{2}\right)+\sum_{j=1}^{p} \sum_{k, k^{\prime}=1}^{n_{i j}} E\left(e_{i j k} e_{i j^{\prime} k^{\prime}}\right)+\sum_{j, j^{\prime}=1}^{p} \sum_{k, k^{\prime}=1}^{n_{i j}} E\left(e_{i j k} e_{i j^{\prime} k^{\prime}}\right)+\sum_{\substack{i=1 \\
i \neq i^{\prime}}}^{m} \sum_{j, j^{\prime}=1}^{p} \sum_{k, k^{\prime}=1}^{n_{i j}} E\left(e_{i j k} e_{i j^{\prime} k^{\prime}}\right]\right. \\
= & \frac{1}{m p^{2} n_{i j}^{2}}\left[p n_{i j} \sigma^{2}+p n_{i j}\left(n_{i j}-1\right) \sigma^{2} \rho_{1}+n_{i j}^{2}(p-1) \sigma^{2} \rho_{2}+(m-1) p^{2} n_{i j}^{2} \sigma^{2} \rho_{3}\right] \\
= & \frac{\sigma^{2}}{m p n_{i j}}\left(1+\left(n_{i j}-1\right) \rho_{1}+(p-1) n_{i j} \rho_{2}+(m-1) p n_{i j} \rho_{3}\right) \\
= & \frac{\sigma^{2}}{m p n_{i j}}\left(1+n_{i j}\left(\rho_{1}-\rho_{2}\right)+p n_{i j}\left(\rho_{2}-\rho_{3}\right)+m p n_{i j} \rho_{3}-\rho_{1}\right)
\end{aligned}
$$

Substituting equation(31a), (31b), (31c) And (31d) in equation (21) we get 


$$
\begin{aligned}
& E(M S A)=\frac{\sum_{i=1}^{m} \sum_{j=1}^{p} n_{i j} \alpha_{i}^{2}}{(m-1)}+\frac{1}{(m-1)} \sum_{i=1}^{m} \sum_{j=1}^{p} n_{i j}\left(C_{1}+C_{2}-2 C_{3}-2 \alpha_{i} C_{1}\right) \\
& \left.=\frac{\sum_{i=1}^{m} \sum_{j=1}^{p} n_{i j} \alpha_{i}^{2}}{(m-1)}+\frac{1}{(m-1)} \sum_{i=1}^{m} \sum_{j=1}^{p} n_{i j}\left[\frac{\sigma^{2}}{p n_{i j}}\left(1-\rho_{1}\right)+n_{i j}\left(\rho_{1}-\rho_{2}\right)+p n_{i j} \rho_{2}\right)+\frac{\sigma^{2}}{m p n_{i j}}\left[1+n_{i j}\left(\rho_{1}-\rho_{2}\right)+m p n_{i j} \rho_{3}-\rho_{3}\right)\right]- \\
& \left.\frac{2 \sigma^{2}}{m p n_{i j}}\left[1+n_{i j}\left(\rho_{1}-\rho_{2}\right)+n_{i j}\left(\rho_{2}-\rho_{3}\right)+m p n_{i j} \rho_{3}-\rho_{1}\right]\right] \\
& \left.=\frac{\sum_{i=1}^{m} \sum_{j=1}^{p} n_{i j} \alpha_{i}^{2}}{m(p-1)}+\frac{\sigma^{2}}{(m-1)}\left(\frac{1}{p}\left(1-\rho_{1}\right)+n_{i j}\left(\rho_{1}-\rho_{2}\right)+p n_{i j} \rho_{2}\right)+\frac{1}{m p}\left[1+n_{i j}\left(\rho_{1}-\rho_{2}\right)+m p n_{i j} \rho_{3}-\rho_{3}\right)\right) \\
& \left.=\frac{\sum_{i=1}^{m} \sum_{j=1}^{p} n_{i j} \alpha_{i}^{2}}{m(p-1)}+\frac{\sigma^{2}}{(m-1)}\left(\frac{1}{p}\left(1-\frac{1}{m}\right)\left(1-\rho_{1}\right)+\frac{n_{i j}}{p}(1-) \frac{1}{m}\right)\left(\rho_{1}-\rho_{2}\right)+n_{i j}\left(1-\frac{1}{m}\right)\left(\rho_{1}-\rho_{2}\right)\right) \\
& =\frac{\sum_{i=1}^{m} \sum_{j=1}^{p} n_{i j} \alpha_{i}^{2}}{(m-1)}+\sigma^{2}\left[\left(1-\rho_{1}\right)+\frac{1}{m} \sum_{i=1}^{m} \sum_{j=1}^{p} n_{i j}\left[\frac{1}{p}\left(\rho_{1}-\rho_{2}\right)+\left(\rho_{2}-\rho_{3}\right)\right]\right]
\end{aligned}
$$

\begin{tabular}{|c|c|c|c|c|c|}
\hline $\begin{array}{l}\text { Sources } \\
\text { of } \\
\text { variation }\end{array}$ & $\begin{array}{l}\text { Degree } \\
\text { of } \\
\text { freedom }\end{array}$ & $\begin{array}{l}\text { Sum of } \\
\text { squares } \\
\text { S.S }\end{array}$ & $\begin{array}{l}\text { Mean } \\
\text { square } \\
\text { M.S }\end{array}$ & \multicolumn{2}{|c|}{$\begin{array}{c}\text { Expected Mean Square } \\
\text { E.M.S }\end{array}$} \\
\hline & & & & For independent Data & For dependent data \\
\hline A & $\mathrm{m}-1$ & $S S A$ & $\frac{S S A}{(m-1)}$ & $\sigma^{2}+\frac{1}{(m-1)} \sum_{i=1}^{m} \sum_{j=1}^{p} n_{i j} \alpha_{i}^{2}$ & $\begin{aligned} & =\frac{\sum_{i=1}^{m} \sum_{j=1}^{p} n_{i j} \alpha_{i}}{(m-1)}+\sigma^{2}\left[\left(1-\rho_{1}\right)+\right. \\
& \left.\frac{1}{m} \sum_{i=1}^{m} \sum_{j=1}^{p} n_{i j}\left[\frac{1}{p}\left(\rho_{1}-\rho_{2}\right)+\left(\rho_{2}-\rho_{3}\right)\right]\right]\end{aligned}$ \\
\hline B & $\mathrm{m}(\mathrm{p}-1)$ & $S S B$ & $\frac{S S B}{m(p-1)}$ & $\sigma^{2}+\frac{1}{m(p-1)} \sum_{i=1}^{m} \sum_{j=1}^{p} n_{i j} \gamma_{i j}^{2}$ & $\frac{\sum_{i=1}^{m} \sum_{j=1}^{p} n_{i j} \gamma_{i j}}{m(p-1)}+\sigma^{2}\left[\left(1-\rho_{1}\right)+\frac{1}{m} \sum_{i=1}^{m} \sum_{j=1}^{p} n_{i j}\left(\rho_{1}-\rho_{2}\right)\right]$ \\
\hline Error & $\mathrm{mp}(\mathrm{N}-1$ & SSe & $\frac{S S e}{m p(N-1)}$ & $\sigma^{2}$ & $\left(1-\rho_{1}\right) \sigma^{2}$ \\
\hline Total & $\mathrm{mpN}-1$ & SST & & & \\
\hline
\end{tabular}

\section{Analysis of variance table}

Analysis of variance table for the model of study, which is given in equation (5), is given in table 1 .

Table 1: Analysis of variance

Where $S S A, S S B, S S e$ and $S S T$ are given in equation (8) 


\section{The $\mathbf{F}$ test for equality of Factor Level Means}

In the last section, we have prepared the ANOVA table, So in this section we will discuss two case for two null hypothesis to know whether the factor level means are equal for the first factor and for the second factor, which is the nested factor. Hence, the alternative conclusions, which we want to, consider for the model of study, are the following .for the first factor is

$$
\begin{aligned}
& H_{\circ}: \alpha_{i}=0 \quad \forall \mathrm{i} \\
& \mathrm{H}_{1}: \alpha_{i} \neq 0 \text { for some } \mathrm{i}
\end{aligned}
$$

And for the nested factor is

$$
\begin{aligned}
& H_{0}: \gamma_{i j}=0 \quad \forall i \text { and } j \\
& H_{1}: \gamma_{i j} \neq 0 \text { for at least one ior } j
\end{aligned}
$$

Because of the additive property for both total sum of squares and degrees of freedom, therefore according to cochrans theorem (if all $\mathrm{n}$ observations $Y_{i}$ come from the same normal distribution with mean $\mu$ and variance $\sigma^{2}$ and sum square total SSTO is decomposed into $k$ sum square $S S r$ and every sum with degree freedom $d f_{r}$ then $S S R / \sigma^{2}$ independent variable distribute $\chi^{2}$ with $d f_{r}$ degree of freedom if $\left.\sum_{r=1}^{k} d f_{r}=n-1\right)$ and under the null hypothesis.

$$
\begin{aligned}
& W_{A}=\frac{S S A}{\sigma^{2}} \sim\left[\left(1-\rho_{1}\right)+\frac{1}{m p} \sum_{i=1}^{m} \sum_{j=1}^{p} n_{i j}\left(\rho_{1}-\rho_{2}\right)\right] \chi_{(m-1)}^{2} \\
& W_{B}=\frac{S S B}{\sigma^{2}} \sim\left[\left(1-\rho_{1}\right)+\frac{1}{m} \sum_{i=1}^{m} \sum_{j=1}^{p} n_{i j}\left[\frac{1}{p}\left(\rho_{1}-\rho_{2}\right)+\left(\rho_{2}-\rho_{3}\right)\right]\right] \chi_{m(p-1)}^{2} \\
& W_{e}=\frac{S S e}{\sigma^{2}} \sim\left(1-\rho_{1}\right) \chi_{m p(N-1)}^{2}
\end{aligned}
$$

Where $W_{A}, W_{B}$ and $W_{e}$ are independent of each other, having chi-square distribution each with respective degrees of freedom $(m-1), m(p-1)$ and $m p(N-1)$. Therefore we can form the $F_{1} *$ statistic for testing the equality of factor level means of the first factor as follows

$$
\begin{aligned}
F_{1} * & =\frac{W_{A}\left[\left(1-\rho_{1}\right)+\frac{1}{m} \sum_{i=1}^{m} \sum_{j=1}^{p} n_{i j}\left[\frac{1}{p}\left(\rho_{1}-\rho_{2}\right)+\left(\rho_{2}-\rho_{3}\right)\right](m-1)\right.}{W_{E} /\left(1-\rho_{1}\right) m p(N-1)} \\
& =C_{1} F_{1}
\end{aligned}
$$

Where

$$
\begin{aligned}
C_{1} & =\frac{\left(1-\rho_{1}\right)}{\left[\left(1-\rho_{1}\right)+\frac{1}{m} \sum_{i=1}^{m} \sum_{j=1}^{p} n_{i j}\left[\frac{1}{p}\left(\rho_{1}-\rho_{2}\right)+\left(\rho_{2}-\rho_{3}\right)\right]\right]} \\
F_{1} & =\frac{M S A}{M S e}
\end{aligned}
$$


Also, we can form the $F_{2} *$ statistic for testing the equality of factor level mean of the nested factor as follows

$$
\begin{aligned}
F_{2} * & =\frac{W_{B}\left[\left(1-\rho_{1}\right)+\frac{1}{m p} \sum_{i=1}^{m} \sum_{j=1}^{p} n_{i j}\left(\rho_{1}-\rho_{2}\right)\right] m(p-1)}{W_{E} /\left(1-\rho_{1}\right) m p(N-1)} \\
= & C_{2} F_{2}
\end{aligned}
$$

Where

$$
\begin{aligned}
C_{2} & =\frac{\left(1-\rho_{1}\right)}{\left[\left(1-\rho_{1}\right)+\frac{1}{m p} \sum_{i=1}^{m} \sum_{j=1}^{p} n_{i j}\left(\rho_{1}-\rho_{2}\right)\right]} \\
F_{2} & =\frac{M S B}{M S e}
\end{aligned}
$$

\section{Correcting for correlation's}

The correcting factor $C_{1}$ and $C_{2}$ which is given in equation (37), (39) could be in the following three cases:

Case 1: the correcting factor $=1$

Case 2: the correcting factor $>1$

Case 3: the correcting factor $<1$

When we are in the first case, then no correction is needed to correct $F$ test . otherwise the correction is essential and we cannot ignore it.

To find the effect of correlation's for testing the factors effects in model (5), the correcting factors $C_{1}$ and $C_{2}$ are calculated for several different values of correlation's $\rho_{1}, \rho_{2}$ and $\rho_{3}$ and also for several different values of $m, p, N$ are shown in tables 2, 3, 4 and 5 respectively.

$$
C_{1}=\frac{\left(1-\rho_{1}\right)}{\left[\left(1-\rho_{1}\right)+\frac{1}{m} \sum_{i=1}^{m} \sum_{j=1}^{p} n_{i j}\left[\frac{1}{p}\left(\rho_{1}-\rho_{2}\right)+\left(\rho_{2}-\rho_{3}\right)\right]\right]}
$$

Table 2: shows the different values of the correcting factor $C_{1}$

\begin{tabular}{|c|c|c|c|c|c|}
\hline & & & $\begin{array}{l}m=2, p=2, N=18 \\
n_{11}=2, n_{12}=3, n_{13}=4 \\
n_{21}=2, n_{22}=3, n_{23}=4\end{array}$ & $\begin{array}{l}m=2, p=2, N=30 \\
n_{11}=14, n_{12}=6 \\
n_{21}=7, n_{22}=3\end{array}$ & $\begin{array}{l}m=3, p=3, N=60 \\
n_{11}=5, n_{12}=6, n_{13}=7 \\
n_{21}=4, n_{22}=6, n_{23}=12 \\
n_{31}=3, n_{32}=9, n_{33}=8\end{array}$ \\
\hline .005 & .05 & $\rho_{2}$ & $\rho_{3}$ & 0.678 & 0.613 \\
.001 & .005 & .002 & 0.985 & 0.869 & 0.75 \\
.005 & .001 & .001 & 0.976 & 0.957 & 0.949 \\
.05 & .05 & .05 & 1 & 1 & 1 \\
.01 & .05 & .055 & 1.2 & 1.467 & 1.588 \\
.01 & .02 & .01 & 1.398 & 2 & 2.75 \\
\hline
\end{tabular}




$$
C_{2}=\frac{\left(1-\rho_{1}\right)}{\left[\left(1-\rho_{1}\right)+\frac{1}{m p} \sum_{i=1}^{m} \sum_{j=1}^{p} n_{i j}\left(\rho_{1}-\rho_{2}\right)\right]}
$$

Table 3: shows the different values of the correcting factor $C_{2}$

\begin{tabular}{|c|c|c|c|c|}
\hline$\rho_{1}$ & $\rho_{2}$ & $\begin{array}{l}m=2, p=2, N=18 \\
n_{11}=2, n_{12}=3, n_{13}=4 \\
n_{21}=2, n_{22}=3, n_{23}=4\end{array}$ & $\begin{array}{l}m=2, p=2, N=30 \\
n_{11}=14, n_{12}=6 \\
n_{21}=7, n_{22}=3\end{array}$ & $\begin{array}{l}m=3, p=3, N=60 \\
n_{11}=5, n_{12}=6, n_{13}=7 \\
n_{21}=4, n_{22}=6, n_{23}=12 \\
n_{31}=3, n_{32}=9, n_{33}=8\end{array}$ \\
\hline .05 & .01 & 0.888 & 0.76 & 0.78 \\
\hline .005 & .003 & 0.994 & 0.985 & 0.987 \\
\hline .05 & .05 & 1 & 1 & 1 \\
\hline .001 & .005 & 1.004 & 1.031 & 1.027 \\
\hline .01 & .05 & 1.138 & 1.435 & 1.369 \\
\hline
\end{tabular}

Table 4: shows the true alpha level for some different values of $C_{1}$

\begin{tabular}{|c|l|l|l|l|l|}
\hline$C_{1}$ & 0.01 & 0.025 & 0.05 & 0.1 & 0.25 \\
\hline 0.4 & 0.056 & 0.109 & 0.19 & 0.348 & 0.408 \\
0.6 & 0.04 & 0.08 & 0.143 & 0.265 & 0.355 \\
0.8 & 0.025 & 0.053 & 0.097 & 0.183 & 0.303 \\
1 & 0.01 & 0.025 & 0.05 & 0.1 & 0.25 \\
1.2 & 0 & 0 & 0.003 & 0.017 & 0.197 \\
1.4 & 0 & 0 & 0 & 0 & 0.145 \\
\hline
\end{tabular}

Table 5: shows the true alpha level for some different values of $C_{2}$

\begin{tabular}{|c|l|l|l|l|l|}
\hline$C_{2}$ & 0.01 & 0.025 & 0.05 & 0.1 & 0.25 \\
\hline 0.4 & 0.044 & 0.087 & 0.155 & 0.294 & 0.361 \\
0.6 & 0.033 & 0.067 & 0.12 & 0.229 & 0.324 \\
0.8 & 0.021 & 0.046 & 0.085 & 0.165 & 0.287 \\
1 & 0.01 & 0.025 & 0.05 & 0.1 & 0.25 \\
1.2 & 0 & 0.002 & 0.011 & 0.029 & 0.209 \\
1.4 & 0 & 0 & 0.004 & 0.008 & 0.173 \\
\hline
\end{tabular}


We can note from table 4,5 , that the true $\alpha$ level in flate /deflate when the correction factor greater/less than 1,and this leads to have a bigger/smaller rejection region for the complete null hypothesis on testing factors. Hence the uncorrected F test can be liberal for some tests and conservative for others in ANOVA when the correlation structure is ignored.

\section{The Relationship between $\alpha$ and true $\alpha$}

The relationship between values of $\alpha$ when data are uncorrelated and values of the true $\alpha$ when data are correlated for different values of the correction factor $C_{1}, C_{2}$ is shown in figure 1, 2 when $m=2, \quad p=2, \quad N=18, n_{11}=2, n_{12}=3, n_{13}=4, n_{21}=2$, $n_{22}=3, n_{23}=4$.

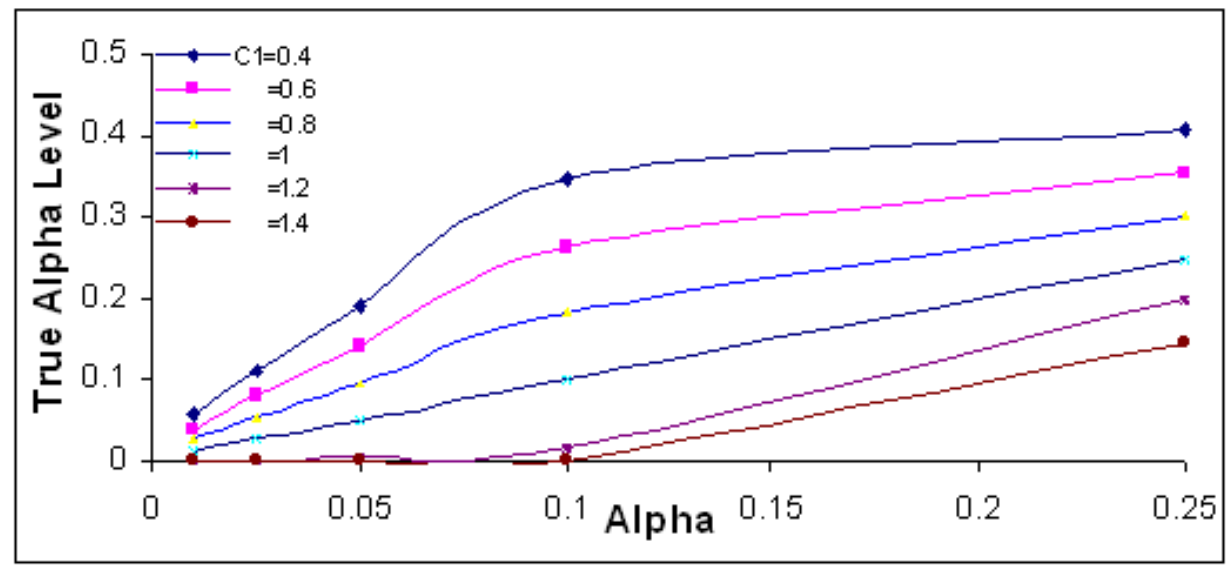

Figure1:True Alpha versus Alpha for different values of $C_{1}$

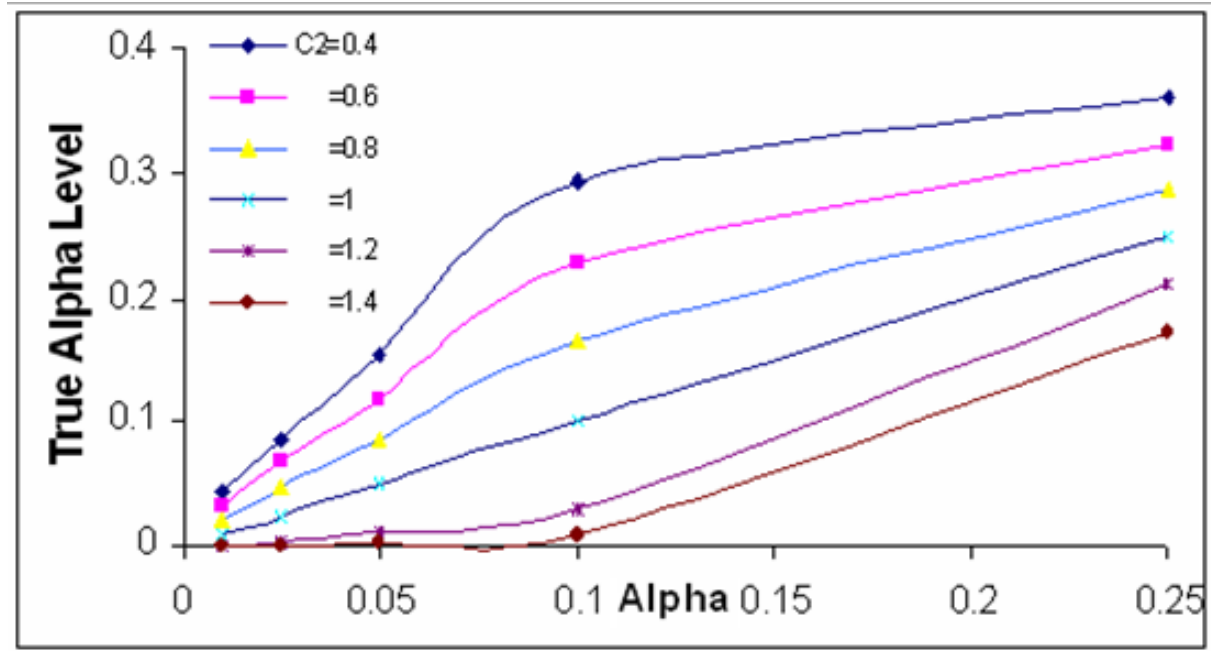

Figure2:True Alpha versus Alpha for different values of $C_{2}$

\section{Conclusion}

Correlation's constant $C_{1}, C_{2}$ small correlation can be amplified by the number of treatment $n_{i j}, i=1,2, . ., m j=1,2, \ldots, p$. And these correlations can thus easily inflate the type I error Rate. Table 1.4, 1.5 show that the true alpha inflate/deflate when the correction constant greater/less than 1,the uncorrected $\mathrm{F}$ test would be liberal test (high 
type I error rate). In conclusion, The $\mathrm{F}$ statistic can be effected by very large correlations sometimes the same as very small correlations. That is, the F statistic is affected by the value of the correction constant $C_{1}, C_{2}$ and not the value of correlations. 


\section{$\underline{\text { REFERENCES }}$}

[1] AL-Rabeaa, Z.A. 1995, "The Effect of Dependent Data on Type I Error Rates for Multiple Comparisons Procedures", M.Sc Thesis, College of Science, University of Basrah.

[2] Arnold, S.F. 1981, "Theory of linear Models and Multivariate Analysis", john Wiley, New York.

[3] Gabbara, S.D. and AL-Rabeaa, Z.A.1996, "The Effect of Dependent Data on Type I Error Rates for Multiple Comparisons Proceduress", Journal of Iraqi Statistical Assocition Proceeding of the $8^{\text {th }}$ conference.

[4] Gastwirth, J.L. and Rubin, H. 1972, "Effect of Dependence of level of Some One- Sample Test”, JASA, Vol. 66, No. 3, PP. 816-820.

[5] Latif, I.S. 2000, "Determining The Expected Mean Squares For 2-way Nested Effects Model With Weak Correlated Data", M.Sc. Thesis, College of Science, University of Salahaddin.

[6] Pavur,R.J. and Davenport,J.M.1985, “ The(Large) Effect of (small) Correlation In ANOVA and Correction Procedures”, Amer.J.Math. \& Manag. Sci.,Vo. 15,No. 2, PP. 77-92.

[7] Pavur, R.J. 1988, "Type I Error Rates for Multiple Comparisons procedures With Dependent Data”, J. Amer. Statis. Assoc., Vol. 42, NO. 3, PP. 171-173.

[8] Smith, J.H. and Lewis, T.O. 1980, "Determining the Effects of Interclass Correlation On Factorial Experiments", J. Amer. Statis. Assoc.,Vol.13, NO.9, PP.1553-1556.

[9] Neter, J. and Wasserman, W. 1974, “Aplied linear Statistical Models”, IRWIN.

[10] Ott, Lyman. 1984, "An introduction to method and data analysis", Duxbury Press. Wadsworth, Inc.

[11] Rao, C.R. and Mitral, S.K. 1971, "Generalized Inverse of Matrices and Its Applications", NewYork: John Wiley and Sons, Inc. Canada. 